\title{
Application of Improved Evidence Theory Algorithm to Health Diagnosis of Mine Belt Conveyors
}

\author{
Ruimin Qi ${ }^{\mathrm{a}}$, Guodong Zhang \\ Zhengzhou University of Industrial Technology College of Mechanical and Electrical \\ Engineering Zhengzhou, China \\ aqrm2007@126.com, bhangguodong1983@126.com
}

Keywords: information fusion, evidence theory, method of improved fusion

Abstract: According to the multi sensors in the actual environment are susceptibly disturbed, and also the traditional evidence theory has the limitations in the data fusion, so this paper presents an improved information fusion method. Information the belt conveyor sensor acquisition is fuzzed, fusion is performed according to a certain proportion, so as to realize the comprehensive judgment in the conveyor performance. Experiments show that the method can improve security and reliability of the conveyor.

\section{Introduction}

Belt conveyor is the key equipment in coal mine transportation. It has taken the place of traditional transportation, for example, train transportation and car transportation. It has realized the mechanization and automation of mining production. But the conveyor line is longer, manual monitoring is difficult, It's not easy to maintain, t's also prone to accidents. Therefore, it is of great significance to ensure the safe and reliable operation of the conveyor.

\section{Existing information fusion algorithm}

Evidence theory can reflect uncertain information, it has a great advantage, it requires evidence to be independent of each other, evidence is equally important. But in practice, each sensor in the system is restricting and influencing, in addition, the accuracy of the sensor is limited. So, evidence can not support each other, the rule of evidence synthesis cannot be used.

\subsection{Method one (evidence theory)}

In information fusion, the core idea of evidence theory (hereinafter referred to as method one) is to obtain the basic probability assignment through multiple sensors, and then merge them according to the synthesis rules, And get the total trust of all the evidence, Suppose that $U$ is an identification framework, Function : $m: 2^{U} \rightarrow[0,1]$ :

$$
\begin{aligned}
& \text { (1) } m(\Phi)=0, \\
& \text { (2) } \sum_{A \subset U} m(A)=1
\end{aligned}
$$

$m(A)$ is the basic probability assignment of $A$, It represents the precise degree of trust in the propositional $A$. The synthesis rule:

$$
m(c)= \begin{cases}\frac{\sum_{A_{\cap} \cap B_{j} \cap \cap_{1} \ldots=C} m_{1}\left(A_{i}\right)^{*} m_{2}\left(B_{j}\right)^{*} m_{3}\left(C_{l}\right) \ldots}{1-k} & \forall C \subset U, \quad C \neq \phi \\ 0 & C=\phi\end{cases}
$$




$$
k=\sum_{A_{i} \cap B_{j} \cap C_{l} \ldots=\varphi} m_{1}\left(A_{i}\right) * m_{2}\left(B_{j}\right) * m_{3}\left(C_{l}\right) \ldots<1
$$

It can be seen from the synthetic formula: when $k<1$, The combination rule can be used; when $k=1$, the combination rule is conflict.It shows that the greater the $\mathrm{K}$, the higher the degree of conflict between the evidence

\subsection{Method two (Paper method)}

Although there is an interaction between the evidences, these values tend to tend to an intermediate value, in order to eliminate the high or low probability of the basic probability assignment caused by these uncertain factors, the scale factor is introduced into the evidence synthesis. Firstly, the coefficient of proportionality in the whole evidence body is determined according to the basic probability assignment:

In information fusion, $n$ sensors provide evidence, and the evidence set is $E=\left\{E_{1}, E_{2}, \ldots, E_{n}\right\}$, in the proof set $E$,it is assumed that the conflict between $E_{i}$ and $E_{j}(j=1,2, \ldots, n)$ is $k_{i j}$, and the collision vector $\mathrm{K}$ is:

$$
\begin{gathered}
K_{i}=\left(k_{i 1}, k_{i 2}, \ldots, k_{i n}\right) \\
k_{i j}=\sum_{\substack{A_{i} \cap A_{j}=\phi \\
A_{i} \in E_{i}, A_{j} \in E_{j}}} m_{i}\left(A_{i}\right) m_{j}\left(A_{j}\right) \text { andi }=1,2, \ldots, n, \text { normalization processing is carried out: } \\
\mathrm{K}_{i}^{\prime}=\frac{\left(k_{i 1}, k_{i 2}, \ldots, k_{i n}\right)}{\sum_{j=1, j \neq i}^{n} k_{i j}}=\left(k_{i 1}^{\prime}, k_{i 2}^{\prime}, \ldots, k_{i n}^{\prime}\right)
\end{gathered}
$$

and the corresponding entropy value is obtained:

$$
H_{i}=\sum_{j=1, j \neq i}^{n} k_{i j}^{\prime} \ln \left(k_{i j}^{\prime}\right)(i=1,2, \ldots, n)
$$

take the $H_{i}$ countdown:

$$
H_{i}^{-1}=\frac{1}{H_{i}}
$$

Suppose that the entropy of evidence $E_{i}$ corresponds to $e_{i}$,

$$
W=\left(e_{1}, e_{2}, \ldots, e_{n}\right)
$$

$e_{i} \in[0,1]$ and $\sum_{i=1}^{n} e_{i}=1$,calculating the entropy of evidence $E_{i}$

$$
e_{i}=\frac{H_{i}^{-1}}{\sum_{j=1}^{n} \frac{1}{H_{j}}}
$$

Then, Take the maximum value in $e_{i}$, it can obtain relative weight vector:

$$
W^{*}=\left(e_{1}, e_{2}, \ldots, e_{n}\right) / e_{\max }=\left(\alpha_{1}, \alpha_{2}, \ldots, \alpha_{n}\right)
$$

According to the principle of the minority to the majority, So $e_{i}$ takes the middle value of $\left\{e_{1}, e_{2} \ldots e_{n}\right\}$, Relative proportionality coefficient: 


$$
W^{*}=\frac{\left(e_{1}, e_{2}, \ldots, e_{n}\right)}{e_{\text {mid }}}=\left(\alpha_{1}, \alpha_{2}, \ldots, \alpha_{n}\right)
$$

When there are even numbers of evidence in the system, $e_{\text {mid }}$ takes the value of $(n+1) / 2$ in $e_{i}$, when there are odd numbers of evidence, take the value of $n / 2$, this can reduce the basic probability of a few information, in order to improve the total support, make the diagnosis more accurate

$$
m_{i}^{*}\left(A_{k}\right)=\alpha_{i} m_{i}\left(A_{k}\right) \quad\left(k=1,2, \ldots, d_{i}\right)
$$

In order to satisfy (2) formula:

$$
m_{i}^{*}(\theta)=1-\sum_{k=1}^{d_{i}} m_{i}^{*}\left(A_{k}\right)
$$

Finally, the basic probability is assigned after the preprocessing, and then the information fusion is carried out according to the synthesis rule of (7):

$$
p(A)=\sum_{A_{i} \cap B_{j} \cap C_{1} \ldots=A} m_{1}^{*}\left(A_{i}\right) * m_{2}^{*}\left(B_{j}\right) * m_{3}^{*}\left(C_{l}\right) \ldots
$$

This method not only modifies the composition rule, but also does the preprocessing of the basic probability assignment. And taking full account of the evidence, the proportion of the whole evidence, so that the information has been more fully utilized.

\section{Example analysis and comparison}

Set identification framework $U=\{A, B\}$, The state of uncertainty is $\Theta$, Six basic probability assignment from the evidence source $S_{1}, S_{2}$, as shown in Table 1:

Table 1 the basic probability assignment of evidence

\begin{tabular}{|c|l|l|c|}
\hline signal source & $A$ & $B$ & $\Theta$ \\
\hline$S_{1}$ & 0.008 & 0.8174 & 0.1746 \\
\hline$S_{2}$ & 0.9999 & 0 & 0.0001 \\
\hline
\end{tabular}

From the table, evidence $S_{1}, S_{2}$ supports model $B$,obviously, the evidence is a high conflict. The four methods are fused as shown in table 2:

Table2 4 fusion results of algorithms

\begin{tabular}{|c|c|c|c|}
\hline & $A$ & $B$ & $\Theta$ \\
\hline Method one & 0.9895 & 0.0104 & 0.0001 \\
\hline Method two & 0.0145 & 0.8545 & 0.1312 \\
\hline
\end{tabular}

It can be seen that method one highly supports $A$ with a probability of 0.9895 , The two method for conflict directly assigned to the empty set (that is, uncertain States), The uncertainty is as high as 0.9995, So that the fusion results can not be obtained, Method three was less supportive, while method four was as high as 0.8545 , Moreover, the uncertainty is low, which makes the judgment of the pattern to be identified more intuitive and accurate.

\section{Conclusions}

To solve the problem of evidence conflict, through the comparison of the four methods, obtained by the improved method of the proportional coefficient makes the conflict part of effective 
allocation, rather than directly assigned to the empty part of the conflict. We can see that the method can effectively distinguish the pattern to be recognized, to demonstrate the accuracy and effectiveness of the method.

\section{References}

[1] Li Haoliang. Bays information fusion based on D-S evidence theory [J]. Fuzzy system and Science,2009 (7) 46-51.

[2] Xu Xiaobin. nformation fusion diagnosis method of random sets based on fuzzy fault feature information[J]. Journal of electronics and information,2009(7):1635-1640.

[3] Basir Otman, Yuan Xiaohong. Engine fault diagnosis based on multi-Sells or information fusion using Dempater-Sharer evidence theory[J]. Information Fusion,2005,25(1):1—8.

[4] Li Wenli, Guo Kaihong. D-S evidence theory synthesis rule and conflict problem [J]. System engineering theory and Practice,2010(8),1422-1432. 